\title{
Estéticas de la inconsistencia en los espacios de la imagen digital
}

\author{
Alejandro Lozano ${ }^{a}$ \\ ${ }^{a}$ Universidad de Salamanca. Contacto: alejandro.Im@usal.es
}

\section{Resumen}

En esta intervención exploro el espacio en el arte digital. Mi punto de partida es la plasticidad de la imagen digital y la afirmación de un primado de la composición espacial que se puede localizar ya desde las primeras prácticas del computer art. Me centro de manera concreta en los espacios tridimensionales navegables, específicamente en el caso de los videojuegos. Los mundos videolúdicos son simulaciones inmersivas, es decir, pretenden envolver al usuario en universos artificiales que puede explorar gracias a la articulación de un conjunto de mecánicas. Los escenarios de juego están diseñados ad hoc para que el jugador interactúe con ellos mediante un proceso de activación y repliegue de sus componentes en función del interés del usuario.

Algunas propuestas, especialmente a partir de la década de 2010, han experimentado con esta flexibilidad del software para crear propuestas que desafían las relaciones convencionales entre sujeto y espacio. Con ello, han generado configuraciones estéticas basadas en la creación de inconsistencias en al menos dos sentidos. El primero de ellos tiene que ver con la explotación de una tensión entre materialidad (desplegarse ante la atención del usuario) e inmaterialidad (replegarse al dejar de ser un elemento de interés) de los elementos interactivos del mundo de juego.

El segundo está relacionado con la incapacidad del jugador para emplear estrategias convencionales de exploración y control del espacio, lo que abre la posibilidad a introducir en la experiencia factores como la vulnerabilidad y la supresión de certezas.

Palabras clave: espacio, digital, videojuegos, inconsistencia, interactividad 


\section{Abstract}

This article explores the space in digital art. I start explaining the plasticity of digital image and the importance that artists have conveyed to spatial composition since the early stages of computer art. I focus on navigable, three-dimensional spaces, and more specifically on video games. Video game worlds are immersive simulations whose purpose is to surround the player in artificial universes through a series of mechanics. Game worlds are designed intentionally so that the player engages with their attractions.

During the decade of 2010, a growing number of video games have experimented with the creation of environments that deviate from conventional relationships between the subject and space, thus creating aesthetics of inconsistency in at least two ways. The first roots in exploiting the tension between materiality (the environment enable attractions for the user) and immateriality (once the user stops paying attention the attractions are no longer active) of interactive components. The second is related to the impossibility of using routine strategies to explore and take control of space, which leads to the possibility of creating experiences of vulnerability and uncertainty.

Keywords: space, digital, video-games, inconsistency, interactivity. 


\section{La maleabilidad de la imagen digital}

Tomaré como punto de partida la maleabilidad de la imagen-código para explicar el concepto de una estética de la inconsistencia en los espacios de la imagen digital ${ }^{1}$. Las aplicaciones de creación y tratamiento de la imagen permiten llevar a cabo todo tipo de operaciones de transformación y mezcla con gran facilidad. Todo proyecto de software está siempre abierto a revisión mediante comandos tan accesibles como deshacer, rehacer, cortar y pegar.

La plasticidad del software es una idea central del computer art desde hace décadas. El Manifesto of Computer Art de Tamas Waliczky (1989) ya identificaba esta cualidad como un componente fundamental del (en aquel entonces) inminente arte digital. Comparadas con las tecnologías de imagen analógica, como un proyector de cine, Waliczky veía en los medios digitales un mayor margen de maniobra para, por ejemplo, romper con la estructura lineal de una proyección fílmica u ordenar aleatoriamente los fotogramas. Una de las piezas tempranas más representativas de este momento seminal del computer art es The Fourth Dimension. Se trata de un trabajo de Zbigniew Rybczynski del año 1988 cuyo efecto consiste en alterar una fina porción del espacio de cada frame de la película con porciones de los frames previos y posteriores. Rybczynski logró dotar de una elasticidad hipnótica los objetos filmados, entre ellos cuerpos humanos.

El efecto óptico al que induce la cuarta dimensión de Rybczynski recuerda al trabajo en un torno de alfarería. La materia pierde su consistencia con cada manipulación de los frames y abre la posibilidad a nuevas formas. Desde 1988 hasta 2019 han transcurrido más de tres décadas, pero podemos aproximarnos a Inmersión de Marina Núñez utilizando este hilo conductor: el espacio fractal que ha creado la artista en esta pieza invita a pensar en un avance incesante hacia un fondo que nunca termina de llegar. Esa sensación de abismo sin límites, con ecos de sublime (Soriano, Sandoval y Núñez, 2019, p. 45), culmina en el encuentro con unas figuras humanas que nos devuelven la mirada y que, a juzgar por la textura de sus cuerpos, están también fundidas con el entorno, como los cuerpos de la Cuarta dimensión de Rybczynski.

Si recuperásemos la tradicional distinción de Lessing entre las artes del tiempo y las artes del espacio, el arte con ordenador pertenecería indudablemente a las segundas. Este primado del espacio lo exploraron primero los artistas en la práctica y con el tiempo fue conceptualizado en la teoría de los nuevos medios. El texto de referencia,

\footnotetext{
${ }^{1}$ Aunque no es estrictamente correcto afirmar que algo compuesto por series de código binario es maleable o dúctil, lo cierto es que estos adjetivos concuerdan con las prácticas de manipulación de imágenes digitales. De hecho, parte del software que se utiliza para crear contenidos en 3D se conoce comúnmente como aplicaciones de modelado.
} 
en este sentido, es The Language of New Media (Manovich, 2001). Manovich afirma allí que en la estética de los medios digitales hay una prevalencia del montaje espacial frente al temporal (Manovich, 2001, pp. 322-326), y con ello recupera las intuiciones del Manifiesto de Tamas Waliczky. Los ordenadores, por así decirlo, ponen las cosas fáciles para jugar dentro de los límites del marco de la imagen, y en ese sentido el peso de la dimensión temporal es menor que, por ejemplo, en el cine. Una investigación posterior de Raquel Santos Ortiz ha refrendado este enfoque. Su análisis de las múltiples técnicas de animación y composición digital apuntan hacia ello: "Si el dispositivo cinematográfico se caracteriza por la "maleabilidad del tiempo" (es decir, el registro puede ralentizarse, acelerarse, invertirse...), la imagen digital presenta una "plasticidad espacial" sin precedentes" (Santos Ortiz, 2013, p. 2).

Una de las variantes del computer art actuales son los videojuegos. Al igual que las piezas de videoarte que hemos mencionado, se producen de manera total o parcial utilizando software de creación y producción audiovisual. La principal diferencia de los videojuegos con las prácticas de videoarte aquí descritas es su naturaleza sistémica, lo que tiene como consecuencia un énfasis en la interactividad y la intervención activa del jugador/espectador (Navarro Remesal, 2016, pp. 28-32). Por otra parte, los videojuegos comparten con el videoarte realizado con ordenador el uso intenso de técnicas composición espacial y el aprovechamiento de la maleabilidad de la imagen código para llevar a cabo sus propuestas. En este sentido, podemos aplicar también a este medio las tesis de Manovich y Santos Ortiz.

Un somero vistazo al estado actual de los videojuegos es botón de muestra suficiente para corroborar la importancia de la composición espacial en su diseño. El "mundo abierto" se ha convertido en un género con entidad propia en plataformas de referencia como Steam (para sistemas PC/Mac/Linux), y durante la década de 2010 se han publicado incontables títulos basados en esta premisa. Espen Aarseth, uno de los pioneros los Game Studies, lo afirmó cuando la disciplina comenzaba su andadura: "El ingenio creativo de los artistas que elaboran las aventuras [quests] de los videojuegos no descansa en su capacidad para crear elementos narrativos [story-building], sino en su diseño del espacio: cómo de bien el camino está camuflado con el paisaje" (Aarseth, 2005, p. 504). Desde entonces han aparecido no pocos trabajos consagrados a analizar la construcción del espacio (y su experiencia) como uno de los principios de la estética de los videojuegos. No extraña, por ello, que se hayan buscado claves de lectura en disciplinas como la arquitectura (Gerber y Götz, 2019) o en el uso de técnicas de perspectiva que podemos encontrar en la pintura de paisajes (Schnaars, 2021, pp. 122125). Tampoco sorprende que se haya identificado una dialéctica muy fructífera entre la agencia del jugador y los intereses de los diseñadores del mundo de juego, sobre todo cuando el primero se resiste a seguir las indicaciones turísticas que los segundos han diseminado por el mapa del mundo (Bonner, 2021). 


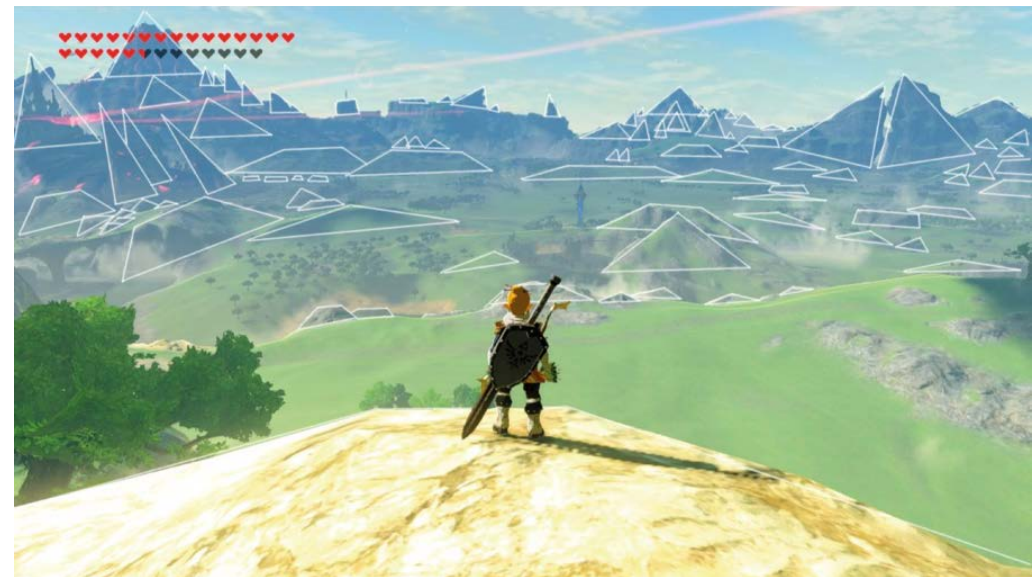

Fig. 1: Composición en triángulos de la perspectiva de THE LEGEND OF ZELDA: BREATH OF THE WILD. Fuente: Schnaars, 2021, p. 123.

\section{Materialidad e inmaterialidad en los espacios de la imagen digital}

Como todo producto de software, un videojuego cuenta con restricciones técnicas. Haciendo de la necesidad virtud, los artistas de este medio han explotado históricamente esas limitaciones como recurso creativo. La primera entrega de la saga de videojuegos de terror psicológico SILENT HILL es un ejemplo ya clásico de este fenómeno (Perron, 2012). El videojuego apareció en 1998 y buena parte de él transcurría en un pueblo ficticio, de modo que esa parte de la acción tenía lugar en exteriores. En la década de 1990, el desarrollo de videojuegos en 3D apenas había despegado y había que afrontar importantes problemas de rendimiento. En el caso de este título se optó por mostrar en pantalla un campo de visión reducido y envolver los alrededores en una densa niebla. Este recurso óptico facilitaba que la videoconsola mostrase menos elementos al jugador $y$, al mismo tiempo, contribuía a generar la atmósfera de terror que requería. Han pasado ya más de dos décadas desde el primer SILENT HILL, pero la gestión de recursos sigue siendo un problema con el que lidiar. La imagen que vemos a continuación muestra cómo en HORIZON: ZERO DAWN se aprovecha el cono de visión de la cámara de juego para discriminar en el nivel de detalle con el que el ordenador procesará los elementos del mundo interactivo. Si el jugador no está mirando una porción del escenario se destinarán menos recursos del sistema para generarla. Este proceso está estandarizado, cuenta con diversas variaciones y se conoce como técnica de culling o desechado. 


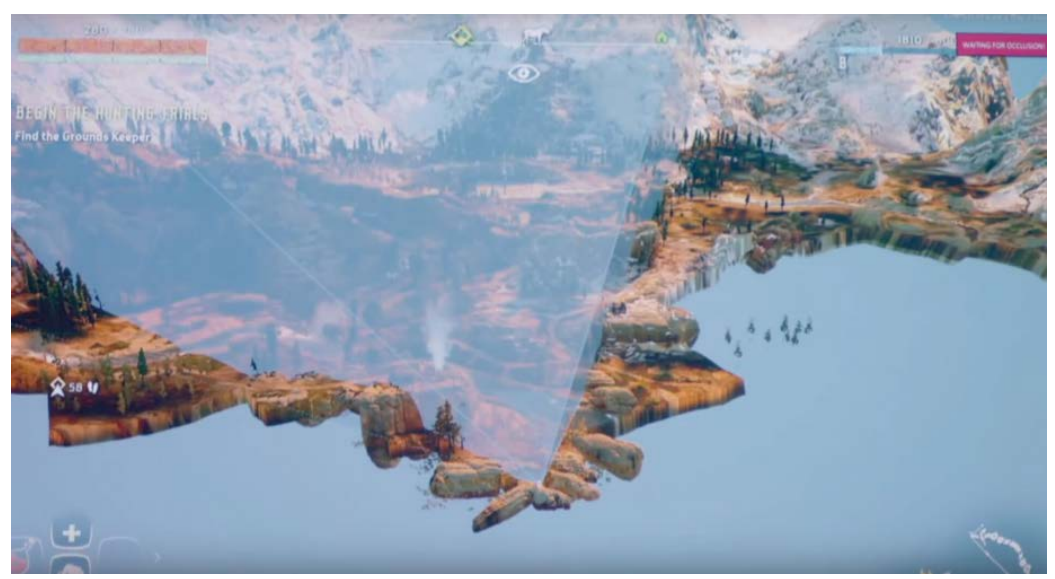

Fig. 2: Cono de visión en HORIZON: ZERO DAWN. Fuente: Bonner, 2021: 69.

Michael Nitsche ha pensado el espacio de los videojuegos como una forma de parque temático (2008, pp. 8-14). La geografía y arquitectura de los mundos videolúdicos se dispone intencionadamente como una serie de atracciones y se persuade al jugador para que se pierda en ellas. Ahora viene bien recordar lo que Baudrillard decía sobre Disneylandia, el padre de todos los parques temáticos, en La precesión de los simulacros (1978): "Todo un abanico de gadgets magnetiza a la multitud canalizándola en flujos dirigidos" (Baudrillard, 2002, p. 25). No olvidemos que, en ese mismo pasaje, el filósofo francés contraponía la experiencia de saturación impostada del interior del parque con la desolación que aguardaba fuera, en el parking ("auténtico campo de concentración"). Aprovecharé esta idea para dar un paso atrás y observar el espacio de los mundos interactivos desde su "aparcamiento".

Lo primero que observamos desde fuera del mundo-diorama es un agudo contraste entre la riqueza del universo interactivo y el abismo sin límites que lo envuelve. Harun Farocki exploró esta tensión original de forma magistral en su serie Parallel (2014), que se compone de 4 piezas en las que reflexiona acerca de diversas implicaciones de la estética del videojuego. Uno de los aspectos sobre los que llama la atención es sobre ausencia de profundidad en el agua de los videojuegos, acerca de la cual escuchamos en Parallel I que "la cámara virtual puede bucear en estas aguas, pero no se ve ninguna corriente oceánica". 


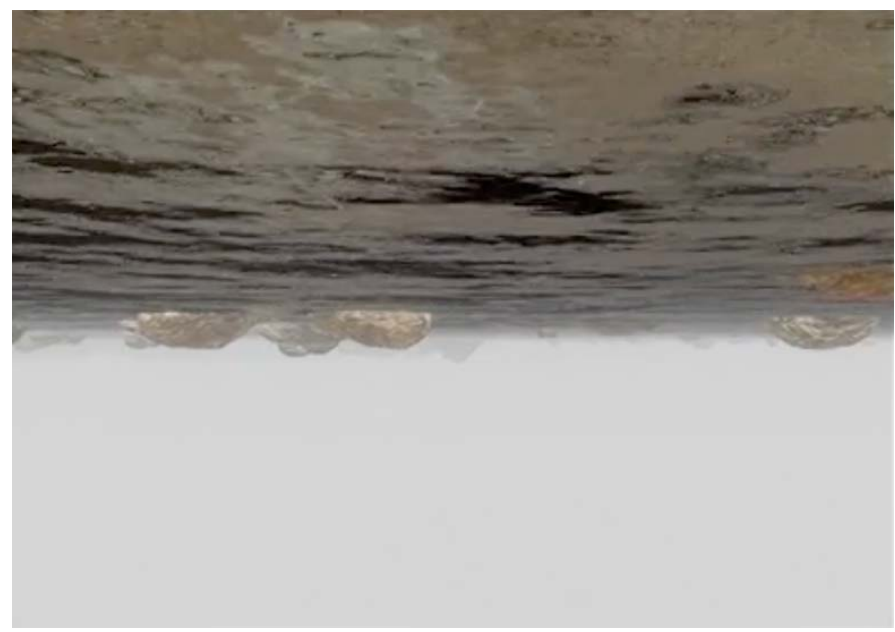

Fig. 3: En Parallel I, la cámara virtual navega bajo el agua en el mundo de juego. Fuente: Apenas Baleia, 2020.

Un producto de software como los videojuegos en 3D solo permite hablar de materialidad e inmaterialidad en sentido figurado, ya que en última instancia estamos hablando de líneas de código que interpretan instrucciones y manipulan ficheros de datos. Pese a todo, este binomio útil para comprender cómo opera el videojuego como un sistema interactivo que reacciona antes los inputs del jugador: el software materializa los componentes y los introduce dentro del sistema de juego cuando es necesario y los elimina cuando han dejado de ser relevantes. En este sentido, el mundo de juego es, primero y ante todo, superficie. Su fina capa material se ha diseñado ad hoc para ofrecer una experiencia de inmersión y está sometida a modificaciones en tiempo de ejecución ${ }^{2}$.

${ }^{2}$ Los videojuegos son sistemas complejos y las interacciones incorrectas pueden provocar fracturas en el tejido del universo lúdico. En ese momento sobreviene el glitch. Un glitch es un tipo de error informático que no llega a provocar un fallo crítico del software, pero que altera la experiencia deseada y los resultados que se obtienen. Una forma de glitch popular se manifiesta como una caída en el abismo. Puede suceder que, debido a algún error en el motor del juego, el personaje del jugador se deslice bajo el suelo y empiece alejarse del escenario indefinidamente, de forma que el escenario original termina convirtiéndose en un punto minúsculo en la lejanía. 


\section{Una estética de la inconsistencia. El caso de P.T.}

Antes de introducir el análisis del caso de estudio es conveniente recapitular la argumentación desarrollada hasta este punto. Al comienzo de esta intervención señalamos que, a lo largo del desarrollo del computer art, tanto la teoría como la práctica han puesto de manifiesto un primado del espacio en la estética de la imagen digital. Ese punto de partida nos ha llevado a explorar la construcción de mundos 3D interactivos en los videojuegos, que son una modalidad actual de arte con ordenadores y hacen uso de este tipo de imágenes. Los videojuegos son sistemas interactivos que construyen y actualizan el mundo lúdico utilizando un procedimiento que puede describirse como una dialéctica entre materialidad e inmaterialidad: la superficie del escenario de juego, el mundo, es como un diorama animado que cobra vida cuando el jugador interactúa con él y se repliega a la mínima expresión cuando deja de llamar la atención del usuario. La conocida afirmación berkeleyana esse est percipi se aplica aquí con rotundidad. Nos encontramos ante una dinámica de actualización de componentes que no solamente tiene alcance técnico, sino que también afecta a la experiencia del jugador $y$, en este sentido, tiene implicaciones desde el punto de vista de la estética.

¿Qué papel desempeña el jugador ante este sistema interactivo? La discusión acerca del tipo de participación de los jugadores en los videojuegos se aleja del propósito de esta intervención. Baste señalar sobre esto que la experiencia del videojuego, en un sentido estético, comprende tanto una dimensión ficcional como otra normativa que articula el conjunto de mecánicas y reglas para dar contexto y sentido a las acciones permitidas (Juul, 2005; Bogost, 2007). En este sentido, el jugador tiene un papel mixto que combina recepción (como lector o espectador de la narración) y agencia (como actor que interactúa con el mundo). La capa ficcional de cada videojuego concreto se puede diferenciar notablemente de otros, pero existen una serie de convenciones de diseño que permiten compararlos desde el punto de vista de su normatividad para establecer diferentes géneros. Dicho de otro modo: cada videojuego construye una ficción distinta, pero desde el punto de vista de sus mecánicas y reglas existen similitudes que permiten establecer diferentes géneros.

Como actores, las tareas que llevan a cabo los jugadores para adaptarse a las peculiaridades de un videojuego de mundo abierto no difieren demasiado de aprender a hacer cualquier tarea rutinaria en otros contextos de la vida. Dinámicas como la repetición de actividades o tomar una ruta concreta para llegar al destino sirven tanto para acomodarnos a una ciudad a la que nos hemos mudado como para adquirir competencias en un nuevo videojuego (Grodal, 2009, p. 148). Al orientarnos y explorar mundos interactivos trasladamos recursos aprendidos en otros contextos de la vida. Sabemos que en un videojuego como GRAND THEFT AUTO podemos ir de un punto a otro del mapa de juego trazando una ruta como lo haríamos para ir a cualquier destino 
real. Lo mismo sucede dentro de edificios o escenarios cerrados en los que subir una escalera siempre lleva hacia un punto más alto del mismo modo que tras una puerta suele haber una habitación. Dicho resumidamente: la interacción con el espacio de un videojuego se basa en una serie de destrezas adquiridas y de expectativas que se basan en ellas.

Algunos videojuegos han experimentado con la dialéctica materialidad-inmaterialidad de los espacios 3D para impactar en las rutinas de exploración de los jugadores. La idea de fondo consistiría en aprovechar que la superficie interactiva del juego es fácil de manipular para conducir al jugador a escenarios en los que se frustran sus expectativas. Aunque hay numerosos ejemplos, me centraré en el caso de P. T. por ser una de las piezas más recientes (a fecha de esta intervención) de la saga SILENT HILL, cuya primera entrega ya he mencionado ${ }^{3}$. P. T. es el acrónimo de Playable Teaser, una demo de 2014 de una entrega de SILENT HILL cuyo producto final nunca se comercializó. Se trata de una producción breve dirigida por Hideo Kojima y Guillermo del Toro que transcurre en el interior de una casa y que, como el resto de las entregas de esta saga, contiene una experiencia de terror de corte psicológico. El jugador debe explorar un pasillo en ' $\mathrm{L}$ ' dispuesto tal y como vemos en la siguiente imagen.

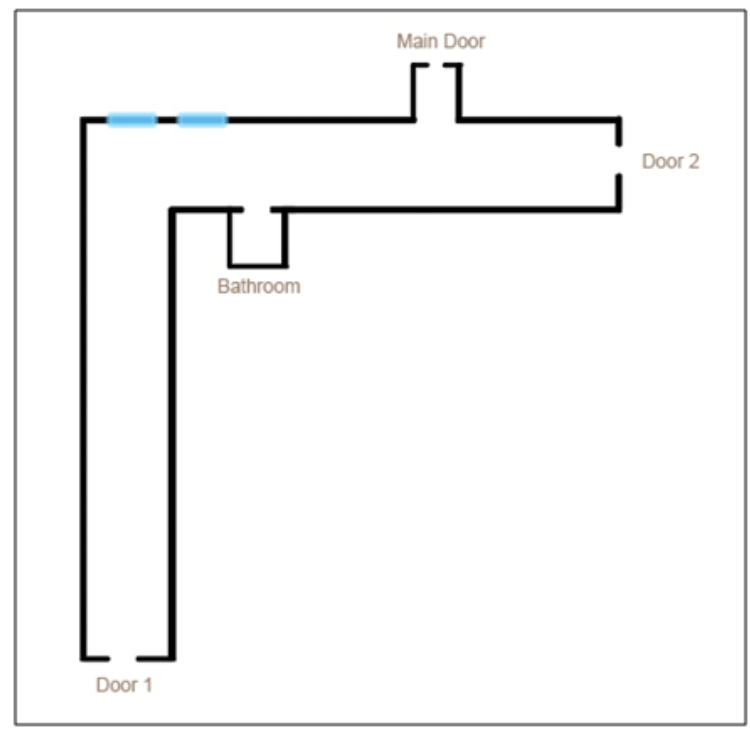

Fig. 4: Escenario de juego de P. T. Fuente: Eisenmann, 2014.

\footnotetext{
${ }^{3}$ Otros videojuegos que han implantado una estrategia similar a P. T. son THE STANLEY PARABLE, ANTICHAMBER, MIND: A PATH TO THALAMUS y THE BEGINNER'S GUIDE.
} 
Tras la puerta 2 se encuentra, sin cortes ni interrupciones, el mismo corredor comenzando por el extremo de la puerta 1 . Este bucle se repite incesantemente a lo largo de toda la pieza. Para avanzar en su desarrollo, es necesario interactuar con algunos elementos en momentos puntuales. A modo de ejemplo, uno de los primeros triggers consiste en mirar un reloj digital al comienzo del pasillo. Con ello se abre la puerta 2 y podemos entrar en un nuevo ciclo del bucle. El jugador tiene poco margen de maniobra, ya que solamente puede recorrer el pasillo y mirar objetos. La atmósfera de la estancia, eventos como cambios en la iluminación y la incapacidad de defenderse ante un eventual ataque contribuyen a crear una experiencia de terror que se basa en buena medida en la vulnerabilidad del personaje. En algunas ocasiones, basta con mirar atrás para morir.

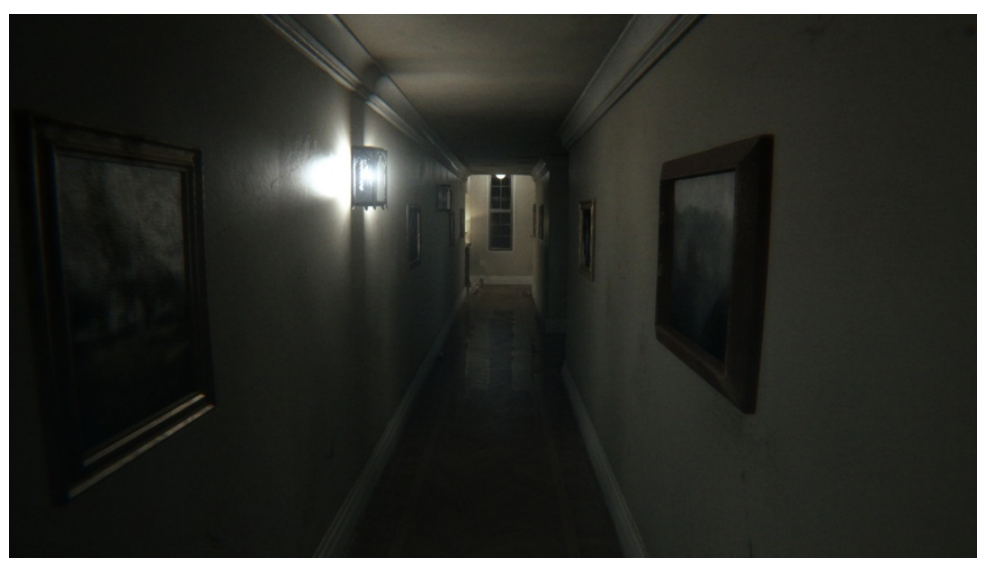

Fig. 5: El pasillo de P. T. en primera persona. Fuente: Silent Hill Wik Español.

Un factor decisivo en esta propuesta es un diseño del espacio inconsistente, en el sentido de que se resiste a entrar en el esquema de lo que aparentemente es el terreno de juego (el pasillo de una casa). Tal y como ha explicado Hans-Joachim Backe, en P. T. fallan incluso estrategias de navegación elementales adquiridas en la infancia, como la permanencia de los objetos (Backe, 2021, pp. 161-164). Al cruzar la puerta 2 la decoración puede haber cambiado, y las únicas explicaciones lógicas desafían a los esquemas espaciotemporales convencionales: o bien hemos caído en un bucle infinito de corredores, o bien los objetos aparecen y desaparecen en el tiempo que tardamos en andar por el pasillo. La experiencia guarda parecido con una visita a las prisiones de Piranesi o los dibujos de Escher, pero con el añadido que conlleva la tercera dimensión (profundidad) y la exploración en primera persona. 


\section{Conclusiones}

Los mundos tridimensionales de los videojuegos son un interesante caso de estudio para explorar las posibilidades estéticas de la imagen digital actual. Basándose en el principio ya clásico del computer art del primado de la composición espacial, este formato pone de relieve una dinámica fructífera facilitada por la maleabilidad del software. El escenario de los mundos de juego está diseñado ad hoc para adaptarse a los intereses del jugador, de forma que el usuario se encuentra en una suerte de parque temático cuyas atracciones se activan o repliegan al instante en función de aquello que llame su atención. Por las características de los universos en 3D, hemos planteado este proceso como una dialéctica entre la materialidad y la inmaterialidad de los componentes de los mundos videolúdicos.

Especialmente durante la década de 2010, algunos videojuegos como P. T. han aprovechado este fenómeno para frustrar estrategias básicas de exploración y gestión del espacio que aprendemos a lo largo de la vida. Este tipo de propuestas configuran una estética de la inconsistencia toda vez que generan una experiencia interactiva en la que el mundo de juego se resiste a una de las expectativas más elementales del jugador: que los objetos tengan consistencia en el espacio y el tiempo.

Este fenómeno invita a pensar que los videojuegos no son solamente un caso de estudio interesante para la estética de las nuevas tecnologías, sino que también pueden tener un impacto en un sentido más amplio. C. Thi Nguyen ha elaborado una argumentación muy convincente para mostrar que los juegos son un arte de agencia: jugando, ya sea en formato analógico o digital, podemos acceder a un catálogo de modos de actuar, asociarnos con otros, establecer metas, etc. (Nguyen, 2020). En este sentido, el juego tendría algún grado de parentesco con las prácticas del arte relacional (Bourriaud, 2008). Una propuesta como la de P. T. podría servir de plataforma para imaginar experiencias en las que se ponen en tela de juicio las relaciones convencionales entre el individuo y el espacio, o, por decirlo en otros términos, entre sujeto y mundo.

\section{Referencias}

Aarseth, E. (2005). From Hunt the Wumpus to EverQuest: Introduction to Quest Theory. En F. Kishino, Y. Kitamura, H. Kato, y N. Nagata (Eds.), Entertainment Computing-ICEC 2005 (pp. 496-506). Springer.

AARSETH, E., y GünzeL, S. (2020). Ludotopia - Spaces, Places, and Territories in Computer Games. Transcript. 
ANTICHAMBER, Demruth (2013). Demruth.

[Apenas Baleia]. (2020). Harun Farocki-Parallel. Vimeo. https://vimeo.com/460744906

BACKE, H. (2021). The Aesthetics of Non-Euclidean Game Spaces. Multistability and Object Permanence in Antichamber and P.T. En M. Bonner (Ed.), Game / World / Architectonics: Transdisciplinary Approaches on Structures and Mechanics, Levels and Spaces, Aesthetics and Perception (pp. 153-167). Heidelberg University Publishing.

BAUDRILLARD, J. (2002). Cultura y simulacro. Kairós.

BEIL, B. (2021). "I love how you can see the bottom of the universe from this room." The RealVirtual Architecture of Davey Wreden's The Beginner's Guide. En M. Bonner (Ed.), Game / World / Architectonics: Transdisciplinary Approaches on Structures and Mechanics, Levels and Spaces, Aesthetics and Perception (pp. 169-185). Heidelberg University Publishing.

Bogost, I. (2007). Persuasive Games. The Expressive Power of Videogames. Cambridge: The MIT Press.

BonneR, M. (2021). The World-Shaped Hall On the Architectonics of the Open World Skybox and the Ideological Implications of the Open World Chronotope. En M. Bonner (Ed.), Game I World / Architectonics: Transdisciplinary Approaches on Structures and Mechanics, Levels and Spaces, Aesthetics and Perception (pp. 65-98). Heidelberg University Publishing.

BourRIAUD, N. (2008). Estética relacional. Adriana Hidalgo.

[Daniele Meringolo]. (2017). The Fourth Dimension (1988) - Zbigniew Rybczynski. YouTube. The Fourth Dimension (1988) - Zbigniew Rybczynski

Dodge, M., Y KITCHIN, R. (2011). Code/Space: Software and Everyday Life. The MIT Press.

EISENMANN, V. (2014). P.T (Silent Hills teaser) game analysis. Gamasutra. https://www.gamasutra.com/blogs/ViktorEisenmann/20140918/225982/PT_Silent_Hill s_teaser_game_analysis.php

Gerber, A., y GötZ, U. (Eds.). (2019). Architectonics of Game Spaces: The Spatial Logic of the Virtual and Its Meaning for the Real. Transcript.

GRAND THEFT AUTO, Rockstar Games (1997 - ). Rockstar Games.

Grodal, T. (2009). Embodied Visions: Evolution, Emotion, Culture, and Film. Oxford University Press.

HORIZON: ZERO DAWN, Guerrilla Games (2017). Sony Interactive Entertainment.

JuUL, J. (2005). Half-Real. Video Games between Real Worlds and Fictional Rules. Cambridge: The MIT Press.

MANOVICH, L. (2001). The Language of New Media. The MIT Press. 
MIND: A PATH TO THALAMUS ENHANCED EDITION. Pantumaca Barcelona (2015). Talking About Media.

Navarro Remesal, V. (2016). Libertad dirigida. Una gramática del análisis y diseño de videojuegos. Santander: Shangrila.

NiTSCHE, M. (2008). Video Game Spaces: Image, Play, and Structure in 3D Worlds. The MIT Press.

Perron, B. (2012). Silent Hill. The Terror Engine. The University of Michigan Press.

P. T., Kojima Productions (2014). Konami.

SAntos, R. (2013). De la maleabilidad del tiempo cinematográfico a la plasticidad del espacio digital. Análisis práctico de construcciones en la imagen código. Tesis doctoral. Universidad de Salamanca. https://gredos.usal.es/handle/10366/122237

SCHNAARS, C. (2021). Taking a Breath of the Wild. The Concept of Airness in Nintendo's take on Open World Games. En M. Bonner (Ed.), Game / World / Architectonics: Transdisciplinary Approaches on Structures and Mechanics, Levels and Spaces, Aesthetics and Perception (pp. 115-132). Heidelberg University Publishing.

SILENT HILL, Konami (1999 - ). Konami.

FANDOM (s.f.). P. T. Silent Hill Wiki Español. https://silenthill.fandom.com/es/wiki/P.T. [Consulta: 28 mayo 2021]

Soriano, D., SANDoval, P., y NuñEZ, M. (2019). Una inmersión con Marina Núñez. En Ayuntamiento de Murcia y Centro Puertas de Castilla (Eds.), Inmersión. Marina Núñez. Murcia.

THE BEGINNER'S GUIDE, Everything Unlimited Ltd. (2015). Everything Unlimited Ltd.

THE LEGEND OF ZELDA: BREATH OF THE WILD, Nintendo (2017). Nintendo.

THE STANLEY PARABLE, Galactic Café (2011). Galactic Café.

WALIZCKY, T. (1989). The Manifesto of Computer Art. Walizcky.net. http://www.waliczky.net/pages/waliczky_manifest_eng.htm 